\title{
Recent advances in gecko adhesion and friction mechanisms and development of gecko-inspired dry adhesive surfaces
}

\author{
Ming ZHOU ${ }^{1, \dagger}$, Noshir PESIKA ${ }^{2}$, Hongbo ZENG ${ }^{3}$, Yu TIAN ${ }^{1, *}$, Jacob ISRAELACHVILI ${ }^{4}$ \\ ${ }^{1}$ State Key Laboratory of Tribology, Tsinghua University, Beijing 100084, China \\ ${ }^{2}$ Chemical and Biomolecular Engineering Department, Tulane University, New Orleans, LA 70118, USA \\ ${ }^{3}$ Department of Chemical and Materials Engineering, University of Alberta, Edmonton, AB, T6G 2V4, Canada \\ ${ }^{4}$ Department of Chemical Engineering, Materials Research Laboratory, University of California, Santa Barbara, California 93106, USA \\ "Present address: Institute of Mechanical Manufacturing Technology, China Academy of Engineering Physics, Mianyang, 621900, China \\ Received: 01 February 2013 / Revised: 10 April 2013 / Accepted: 20 May 2013
}

C The author(s) 2013. This article is published with open access at Springerlink.com

\begin{abstract}
The remarkable ability of geckos to climb and run rapidly on walls and ceilings has recently received considerable interest from many researchers. Significant progress has been made in understanding the attachment and detachment mechanisms and the fabrication of articulated gecko-inspired adhesives and structured surfaces. This article reviews the direct experiments that have investigated the properties of gecko hierarchical structures, i.e., the feet, toes, setae, and spatulae, and the corresponding models to ascertain the mechanical principles involved. Included in this review are reports on gecko-inspired surfaces and structures with strong adhesion forces, high ratios of adhesion and friction forces, anisotropic hierarchical structures that give rise to directional adhesion and friction, and "intelligent" attachment and detachment motions.
\end{abstract}

Keywords: gecko feet surfaces; setae; spatulae; anisotropic dry adhesion and friction; articulated motion

\section{Introduction}

For millennia, the gecko has been well-known for its fantastic climbing abilities. By using only one toe, geckos can easily hang vertically even upside-down from hydrophilic or hydrophobic, smooth or rough surfaces on walls (they cannot hang from a ceiling with only one toe). The typical step intervals of geckos are approximately several tens of milliseconds [1]. Gecko setal arrays have the excellent ability of self-cleaning [2], which has a considerably wider potential application than pressure-sensitive adhesives (PSA) in several areas, such as robotics for rescue and detection, counterterrorism, chemical sensing, and space positioning.

A considerable number of studies have been devoted to understanding the interrelated "frictional adhesion" properties and mechanisms of gecko feet, hairs, and

* Corresponding author: Yu TIAN.

E-mail: tianyu@mail.tsinghua.edu.cn setae in order to mimic the gecko's swift movement on walls and ceilings. Wall-climbing robots based on gecko-inspired adhesives have several advantages over those based on vacuum suction, magnetic adsorption, or velcro, such as small size, flexible and controllable articulation capability, self-cleaning property, and adaptability on rough surfaces [3-4]. Therefore, research on the attachment and detachment mechanisms of gecko feet and hairs and the overall design of gecko-inspired adhesives is of great interest for both theoretical and practical applications on special functional surfaces, articulated robots, and related devices.

In this article, we review the recent advances in gecko adhesion and friction mechanisms and the development of gecko-inspired dry adhesive surfaces. The essential geometric and mechanical properties of the gecko adhesive system are first presented, followed by an overview of the fundamental modeling and understanding of the scientific principles of the gecko adhesive system from the nano-scale contacts 
to the micro-scale structures, to the macro-scale feet and the entire animal. On the basis of the abovementioned understanding, the design principles of gecko-inspired dry adhesive surfaces are identified and the information they reveal about future fabrication strategies is assessed. Finally, we discuss some future issues in this field.

\section{Adhesion and friction mechanisms of gecko seta}

\subsection{Origin and measurement of adhesion forces of gecko seta}

Scanning electron microscopy has enabled the examination of the fine, hierarchical structures of setae. There are approximately twenty rows of lamellas on each gecko toe and approximately 20 setal arrays on each lamella. The single seta is approximately $110-\mu \mathrm{m}$ long and has a diameter of approximately $4-6 \mu \mathrm{m}$ [5]. The seta bifurcates into approximately 100-1000 spatulae at the terminal end [6]. This branched structure ensures close contact between the setae and the (microscopically rough) surfaces that the gecko climbs.

Researchers have proposed several hypotheses to explain the origin of the strong adhesion (and friction) forces of gecko seta, such as electrostatic interaction, vacuum (or suction), microinterlocking (similar to velcro), and glue, which were all eventually disproved by experiments $[7,8]$. The van der Waals and capillary forces are the two major basic interactions between the setae and the surface. Experimental results have indicated that the van der Waals force between the setae and the substrate is the main contributor to the adhesion force, and that the presence of water vapor in the environment may enhance this force through an additional capillary force [9-14]. Similar adhesion forces were observed by Autumn et al. [9] on both hydrophobic and hydrophilic surfaces, which provided the direct evidence for the van der Waals mechanism. Then, many researchers found the enhanced adhesion forces with increasing humidity [10, 11, 15]. Huber et al. [11] also measured the gap between the spatula and the substrate by using white light interferometry, which is only $1-2$ water monolayers even at high humidity. Therefore, the authors proposed that the presence of a monolayer or two of water would modify the van der Waals Hamaker constant. Similarly, Pesika et al. [16] investigated that the surface hydrophobicity of a gecko setal array changed after prolonged exposure to water by using a surface force apparatus (SFA). Further, Puthoff et al. contradicted a capillary mechanism and discovered that an increase in humidity softens the setal $\beta$-keratin, leading to an increase in the adhesion forces [17].

Experimental measurements of the adhesion and friction forces of the hierarchical structures of gecko seta have been performed over the past few years. Hansen and Autumn found that the friction force of an isolated setal array is $0.37 \mathrm{~N}$, with an apparent contact area of $\sim 1.0 \mathrm{~mm}^{2}$, whereas that of a single toe is $4.3 \mathrm{~N}$, with an apparent contact area of $\sim 200 \mathrm{~mm}^{2}$ $\left(2 \mathrm{~cm}^{2}\right)$ The maximum friction force of a single seta (with approximately $100-1,000$ spatulae) is approximately $200 \mu \mathrm{N}$, while the adhesive forces ranges from 20 to $40 \mu \mathrm{N}$. A single seta will spontaneously detach from its opposing surface when the setae shaft subtends an angle of $30^{\circ}$ with the opposing surface [18]. Huber et al. [19] glued an isolated seta perpendicular to the end of an atomic force microscopy (AFM) cantilever, cut most of the terminal branches of the seta away to isolate a few single spatulae, and measured the adhesion force of a single spatula to be approximately $10 \mathrm{nN}$.

The abovementioned experimental friction or shear stresses at different structural levels (i.e., from a single spatula to an entire toe) varied from several kilopascals to $\sim 1,000 \mathrm{kPa}$ because the real contact area was significantly influenced by the preload, test conditions and the nature of the seta arrays [20-22]. These fundamental experiments laid the foundation of subsequent theoretical analyses.

\subsection{Adhesion model of gecko seta}

Along with the experimental studies, significant effort has been made to theoretically analyze the adhesion (and friction) properties of gecko setae and spatulae (see Fig. 1). Since the traditional Hertz model fails to include the surface adhesion between two contacted surfaces, the JKR model of "contact mechanics" or "adhesion mechanics" [23], which considers the force 


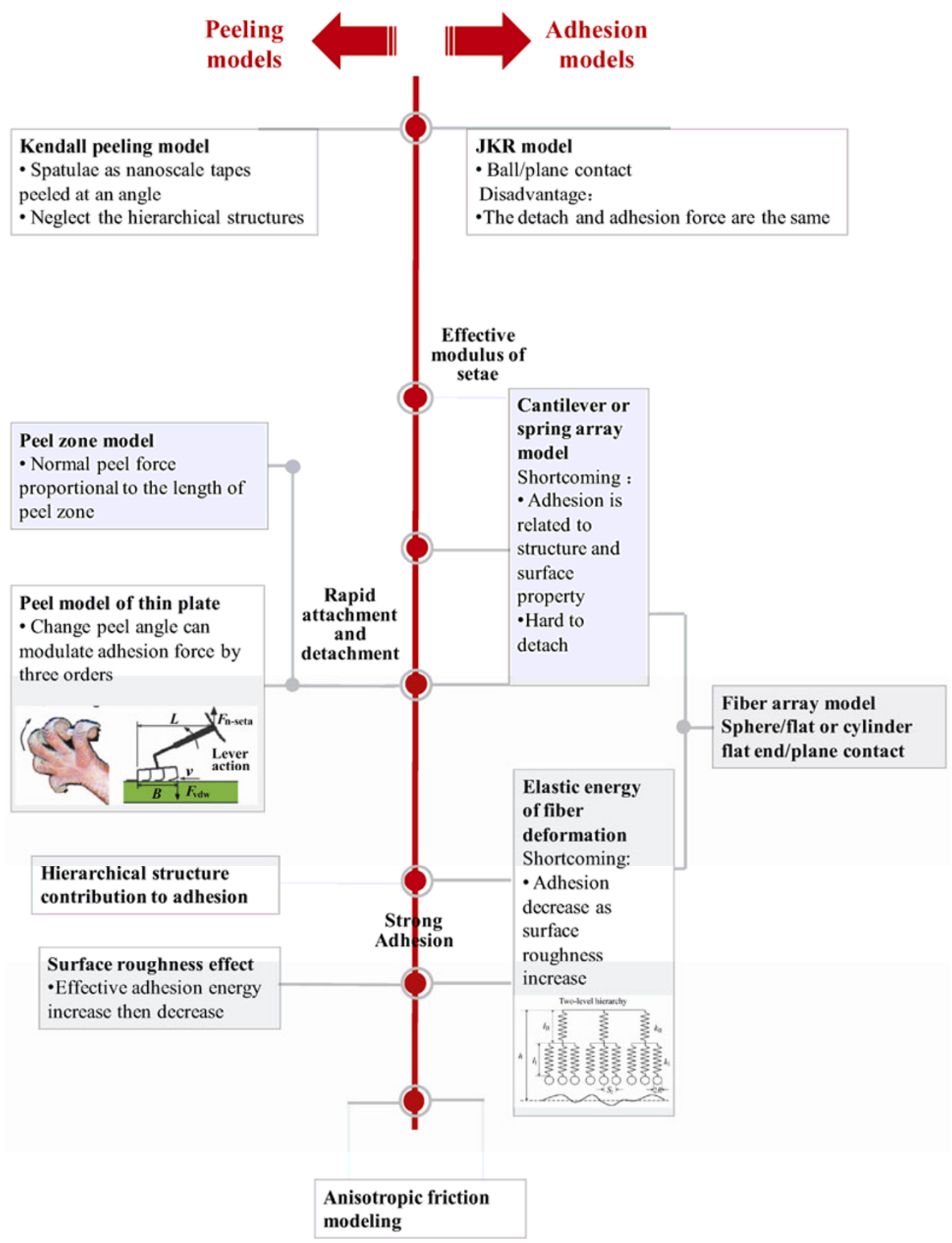

Fig. 1 Theoretical development of adhesion mechanism of gecko seta.

required to pull an elastic sphere of radius $R$ from a planar surface, is used for describing the adhesion force of the gecko seta and spatulae. The gecko spatulae are simplified as cylinders, each terminated with a hemispherical end of radius $R$. Using the adhesion forces measured for a single spatula, we calculated the size of the equivalent JKR sphere to be approximately
$0.13 \mu \mathrm{m}$, which is close to the real (imaged) size of a spatula [11]. The principle of "contact splitting" for bio-inspired fibrillar surfaces has been identified [24, 25], i.e., the adhesive stress of a biological system is inversely proportional to the radius of the terminal structure of the attachment hairs, $R$. Since the adhesion force is proportional to $R$ while the density of contacts 
is proportional to $1 / R^{2}$ based on the self-similar scaling assumption, the total force per unit area is inversely proportional to $R$. Thus, animals with a larger mass usually have smaller attachment structures [25]. However, a more detailed research by Peattie and Full [26] proposed that this scaling law was invalid in phylogenetic relationships between species, which showed that the dynamic properties and the synergistic effect of all hierarchical elements, not just the static contact, should be considered.

One of the disadvantages of applying the JKR model to describe the adhesion of gecko hairs is that the influence of sliding deformation on the enhancement of the adhesion force cannot be explained [27]. How does the sliding influence the contact between the terminal end structure of the seta and the substrate? To address these problems, researchers have developed a fibrillar adhesion model to consider the bending deformation of the setal array during a normal loading process [28]. First, the low effective elastic modulus of the seta array must be understood and theoretically (quantitatively) described: the gecko seta is made of $\beta$-keratin, which has a bulk Young's modulus between 1.3 and $2.5 \mathrm{GPa}$ based on measurements of claws and feathers [29]. Peattie et al. [30, 31] used a resonance technique to directly measure the Young's modulus and found it to lie between 1.4 and 1.6 GPa. The complex modulus of the setal $\beta$-keratin was measured to be 1-4 GPa depending on the environmental relative humidity $[12,26]$. The $103-104-\mathrm{kPa}$ effective elastic modulus of the setal array is significantly lower [32]. Persson and Autumn modeled the relationship between the effective elasticity of fiberarray systems and the bulk materials [32, 33], and Schubert et al. [34] presented a similar analysis. Their experimental and theoretical results explained the low effective elastic modulus of the gecko setal array and showed that this allows for good adaptability for making good contacts on rough surfaces, while the high bulk modulus of $\beta$-keratin itself prevents the self-matting of the neighboring setae, provides an efficient self-cleaning mechanism, and is fractureresistant under high stress. For more information about the formulae, please refer to Ref. [35].

Second, the influence of bending deformations on adhesion has been analyzed by two types of fibrillar adhesion models. In the first type of model, the adhesion force is described as the summation of the adhesion forces of inclined cantilever beams or a spring array of supported hemispheres on a surface [36-38]. The second model shows that the hierarchical structures can increase their effective adhesion energy during detachment from a rough surface by increasing the elastic deformation energy $[33,39]$.

Further, Gao et al. [40] analyzed the detachment of a single seta using a finite element analysis method and proposed that the peel strength can vary by one order of magnitude as a function of the peel angle. The results showed that the adhesion force decreased with a decrease in the peel angle to below $30^{\circ}$ and that the maximum adhesion force was achieved at a peel angle of $30^{\circ}$. However, these theoretical findings did not agree with the experimental results, which showed that a single seta had a lower adhesion force and that it spontaneously detached from a surface at a $30^{\circ}$ tilt angle rather than a smaller tilt angle.

Other researchers have also analyzed the contribution of the hierarchical structures to the adhesion energy or force [40-45]. It was reported that the adhesion strength can change by two orders of magnitude at different tilt angles of the fiber arrays [46, 47]. However, these models focused on the deformation of the upper supporting structures and did not consider the actual contact shape of the terminal structures (the adhesion junctions).

The developed fibrillar adhesion models provide a good understanding of the strong adhesion forces of gecko setal arrays. However, based on the JKR and most fibrillar models, the pull-off force (the maximum force which can be provided by the adhesion interface) and the adhesion force (the critical force to separate the two adhered objects) have the same magnitude, depending only on the material properties and geometric structures of the fiber array. Thus, these models cannot explain how the gecko can quickly detach from surfaces. Moreover, on the basis of these models, the effective adhesion force of the setal array ought to decrease with an increase in the surface roughness. In real situations, the adhesion force of the setae of gecko, flies, and bees initially decreases and then, increases with an increase in the surface roughness $[48,49]$. The minimum adhesion force of the gecko 
spatulae is found on a surface with a roughness of approximately $200 \mathrm{~nm}$, which matches one of the characteristic dimensions of the spatula. Since fibrillar models do not consider the terminal structure of the setae, they cannot fully capture the "contact mechanics" or "adhesion mechanics" of setal arrays.

\subsection{Peeling model of gecko spatulae}

The adhesion models based on fiber arrays described above do not provide an insight into why the detachment force is considerably less than the adhesion force due to the simplification of the terminal structures of setae as simple spheres or flat-ended cylinders, which is very different from the actual thin fan shape of the spatula pad. Therefore, various "peeling models" have been developed to more appropriately demonstrate the mechanism of gecko spatulae detachment, which is analogous to the peeling of adhesive tapes.

The Kendall model [50] describes the peeling strength of an adhesive tape as a function of the peel angle. The spatula pad is simplified as a single strip of tape $[19,20]$ with nanoscale dimensions. The Young's modulus of the spatula is $\sim 2 \mathrm{GPa}$; thus, the elastic energy term in the peeling spatula can be neglected, so that $w=F / \gamma$, where $F=10 \mathrm{nN}$ is the experimental adhesion force of the spatula and $\gamma=50 \mathrm{~mJ} / \mathrm{m}^{2}$. The width of the spatula $w$ is $200 \mathrm{~nm}$, which is close to the actual geometric dimension of the spatula. The Kendall model has provided new insights into the peeling mechanism of gecko detachment [51, 52]. It is also important to note that the biomechanics of a gecko walking on a surface reveals the use of a particular configuration, a Y-shaped geometry/configuration $[1,20,52]$. In this configuration, in order to take a step forward, the gecko always has two diagonally opposite feet on the surface while detaching the other two. The two attached feet are angled to the surface at a certain angle, with a tension along the feet, forming a Y-shaped geometry and yielding a total force in the normal direction to the surface. Further, one foot always has five toes gripped in at different directions. The lateral friction forces due to the toes and feet are finally equilibrated with each other in a static staying sate of the gecko or provide some net friction force to drive the motion of the body.

An understanding of the mechanical behavior of pressure-sensitive adhesive tapes is important to describe the peeling mechanism of the gecko spatula [53-55]. Considering the hierarchical structures of the setae and the macroscopic articulations of gecko toes, particularly how the friction force contributes to the adhesion force, Tian et al. [56] theoretically analyzed the friction and adhesion behavior of gecko pads on the basis of tape peeling model, as shown in Fig. 2(a). High adhesion and friction forces are predicted in the "toe-gripping" actuation, while small release forces are predicted in the "toe-releasing" actuation, these two being determined by the different "peel angles," for gripping and releasing. The lateral friction force and the normal adhesion force of a single seta can change

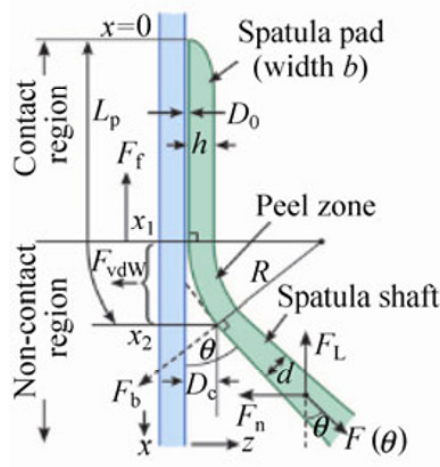

(a)

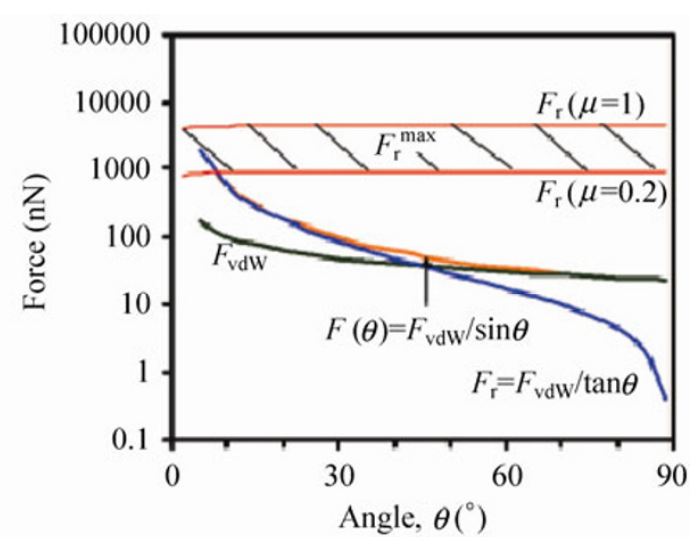

(b)

Fig. 2 (a) Tape model considering the final two levels of the hierarchical structures of setae. (b) Theoretical normal, lateral, and stretching forces of a single spatula at different pulling angles. $\mu$ is the friction coefficient between spatula pad and substrate. In the gripping direction, the peel angle of a spatula pad is decreased in order to approach $0^{\circ}$, while in the releasing direction, the peel angle of the spatula pad is close to or more than $90^{\circ}$. 
by more than three orders of magnitude during gecko toe gripping (attachment) and releasing (detachment), as shown in Fig. 2(b). Using a finite element model, Peng et al. [51] analyzed the change in the peel zone length and the peel force at different peel angles, Young's moduli, and spatula thicknesses. Endlein et al. found that the adhesion forces of tree frogs can also be explained by the peeling theory [57].

Peeling models provide a good way to explain the experimental results of the adhesion forces of gecko setae on surfaces with varying roughness. Fuller and Tabor [58] developed a contact model for elastic solids to describe the effect of roughness in reducing the adhesion force as the real contact area decreases with increasing surface roughness. However, surface roughness can also increase the real contact area of a highly compliant film, leading to the opposite result [59]. Persson and Gorb proposed a qualitative analysis of the effect of the elastic deformation of a spatula on the effective adhesion energy [60]. Peng and Chen [61] demonstrated that the normal adhesion force is dependent on the dimensions of the film with respect to the wavelength of the (sinusoidal) roughness of the substrate.

The developed peeling models provide a good theoretical basis to explain the fundamental mechanisms of seta detachment and the peeling behavior of a single gecko spatula on surfaces with different roughness. Future work can be conducted on the more complex peeling behaviors of hierarchical structures taking into consideration the different geometries and deformations of the spatulae, setae, toe pads, and feet at different length scales.

\subsection{Coupling of friction and adhesion}

Experimental results show that the friction force of gecko setae during sliding along the setal curvature, the gripping in this direction, is considerably higher than the adhesion force, and that lateral sliding is necessary to generate the strong adhesion (and friction) forces $[18,20,27]$. The coupling of the adhesion and friction forces, known as "frictional-adhesion" is one of the most important mechanical properties of the seta $[12,62,63]$.

Setal arrays usually show strong friction anisotropy depending on the shear direction. Different effective elastic moduli of the setae arrays are observed in the loading and unloading force-distance curves when sliding along or against the seta tilt directions (correspond to the gripping in and releasing directions, respectively) [32]. Furthermore, friction and adhesion forces obey different rules in the different sliding directions. The friction force is more than four times larger than the preload when sliding along the gripping direction $[20,21,64]$, and the adhesion force is enhanced. In contrast, when the setal arrays are dragged against the gripping direction, the friction force is less than the preload and obeys Coulomb's law, where the normal force becomes repulsive [20, 21]. These anisotropic properties of the gecko setae are attributed to the anisotropic structure and deformations when the setae or toes are slid or articulated in different directions $[12,65]$.

The coupling of friction and adhesion forces of the setae is significantly influenced by the applied preload. Wan et al. [66] experimentally showed that the preload can decrease the tilt angle of the seta and increase the contact number of spatulae, thereby increasing both the adhesion and the friction forces. However, the normal adhesion force turns into a repulsive force when the preload is above some critical value. Ideally, when the adhesion and friction forces are maximum, the tilt angle is small [67]. However, crowding considerations impose a limit on how small the tilt angle can be before the fibers become overcrowded. A theoretical limiting tilt angle of approximately $12.6^{\circ}$ is consistent with the experimental compression data [68].

The strong anisotropy and synergy between the friction and adhesion performances of the setae arise from the anisotropic deformations of the structures. The friction and adhesion of single seta can reach a stable steady-state value after sliding for several micrometers [18], whereas for the entire setal array, the critical sliding distance was found to be several hundred micrometers. Numerical simulations show that sliding causes the spatulae to become well-aligned or ordered, leading to an increase in the real contact area and to a more stable configuration during sliding $[69,70]$. Cheng et al. [71, 72] proposed that a pre-tension can increase the adhesion force of the seta at small peeling angles. 


\section{Gecko-inspired adhesives}

\subsection{Design principles}

The gecko seta's advanced performance in terms of friction and adhesion endows the gecko with excellent climbing abilities. The creation of a new type of dry adhesive inspired by the gecko adhesive system has received considerable attention. Understanding the key properties, principles, and mechanisms of the gecko adhesive system is essential for the design of bio-inspired dry adhesive surfaces [73].

As discussed above, the "contact-splitting principle" has been recognized and widely accepted [24, 25, 74-78]. On the basis of the JKR model predictions, it can be said that the higher the extent of splitting at the end of the setae, the higher is the adhesion force and the better is the resistance to damage. For these reasons, fibrillar surfaces are now widely used for producing smart adhesive systems. Theoretical analysis shows that fibrillar ends are not sensitive to defects when the size of the fibrils is less than some critical length scale [79]. However, a recent study also shows that the adhesion force does not increase when the number of contact elements increases while the total contact area is constant [80].

Since the mechanical performance is highly dependent on the structures, the size and shape of the optimum fibers is widely discussed. The effect of the shape of the terminal ends becomes more important with increasing size and stiffness of the materials [79]. Spolenak et al. [81] proposed that a flat punch is the perfect shape for a bio-inspired surface, but in practical applications, the properties of the fiber array with a flat punch end may be more easily affected adversely by surface roughness and surface (particulate) contaminants. Gorb and Varenberg [82] proposed that fibers with narrow necks and thin plate-shaped (or mushroom-shaped) ends should be used for overcoming these disadvantages. Experimental results also showed that mushroom-shaped fibril ends perform well during loading-unloading cycles, with improved robustness and stability [83].

An anisotropic structure for the fiber arrays is particularly important in the design of bio-inspired surfaces [84, 85]. Directionally angled polymer flaps were first introduced in the fabrication of a gecko- inspired dry adhesive surface [86]. Basically, the anisotropic behavior of the gecko setae is due to the asymmetric deformation and contacts. Therefore, the asymmetric mechanical designs, including asymmetric shapes [87] as well as different elastic moduli [88] for the fibrillar structures are expected to provide the best prospects for creating the desired performance (for energy-efficient wall climbing, ceiling running, etc.). Therefore, an anisotropic articulation is required to make full use of these anisotropic structures.

A fiber array with a high aspect ratio promotes contact adaptability; however, it should also be noted that slender fibers can easily adhere to neighboring fibers through van der Waals forces, leading to a failure of the device (due to the so-called "crowding" or "bunching" behavior) [89]. Sitti et al. and Hui et al. [40, 90-92] proposed anti-self-adhesion models based on a force and energy analysis, respectively.

Based on an understanding of the above-mentioned design principles, some general design criteria have been developed: The geometric parameters should be designed taking into account the modulus of the materials. Using numerical calculations of a fiber array squeezed by a sphere, Aksak et al. [37] designed the optimum length and diameter of inclined or perpendicular fibers. Spolenak et al. [93] proposed some general "adhesion maps" for fiber arrays with hemisphere-shaped ends, including considerations of condensation, adaptability, contact strength, and fiber fracture, as shown in Fig. 3(a). The target optimum areas are a series of triangles in the map of fibril radius and Young's modulus. Greiner et al. [94] further developed adhesion design maps for fiber arrays with different shapes. Recently, Zhou et al. [95] developed a numerical peel-zone calculation method and proposed an adhesion and peeling design map to evaluate the design criteria for strong attachment and easy detachment (peeling) forces, as shown in Fig. 3(b). The peeling force can be changed by three orders of magnitude with respect to the normal adhesion force by changing the design parameters of the structures.

\subsection{Fabrication}

\subsubsection{Fabrication of gecko-inspired surfaces with strong adhesion}

Based on the original design principle of fiber splitting, 

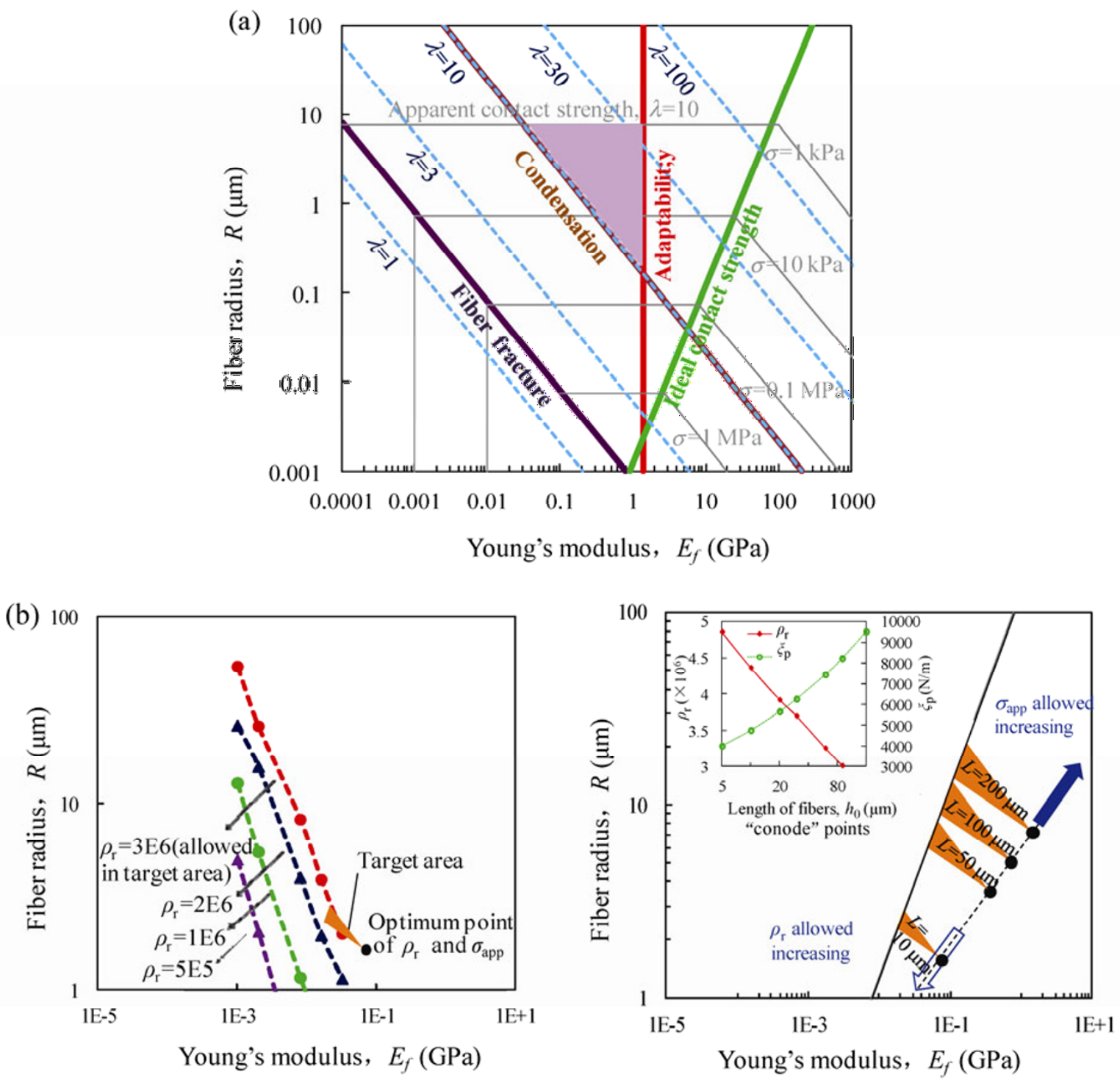

Fig. 3 Design maps of gecko-inspired fibrillar surfaces. (a) Adhesion design maps for gecko-inspired fibrillar surfaces with hemisphereshaped ends. The triangle denotes the target area of $\lambda=10$ (reproduced from Ref. [93]). (b) Adhesion and peeling design map for gecko-inspired fibrillar surfaces with flat ends with the typical values given in Ref. [95]. $\rho_{\mathrm{r}}$ denotes $\rho$ per length at the peel angle of $90^{\circ}$, and $\rho$ is defined as the ratio of the normal adhesion force per unit width (pull-off strength) to the peel strength, which represents the strong attachment and easy-removal properties of surfaces.

microfibrillar surfaces using microfabricated templates with whole arrays and nanowire surfaces with relatively large aspect ratios and high Young's modulus have been fabricated. With further advancements in design principles, fibrillar gecko-inspired surfaces could be developed ranging from simple perpendicular standing sphere-ended single-level fiber arrays to different end-shaped fibers, inclined fiber arrays, fibers with surface modifications, and hierarchical structures, as shown in Fig. 4.
On the basis of the "contact-splitting principle", most of the early publications reported fiber array surfaces with flat or semisphere ends. In the first trial of a templated fabrication, an AFM pin was used for making holes on a wax surface $[89,92]$. With the development of etching technology [25], particularly lithographic techniques, the morphology of the fibers could be perfectly controlled, leading to an increase in adhesion strength [90, 96-100]. Nanowires with self-cleaning properties have been reported, which 


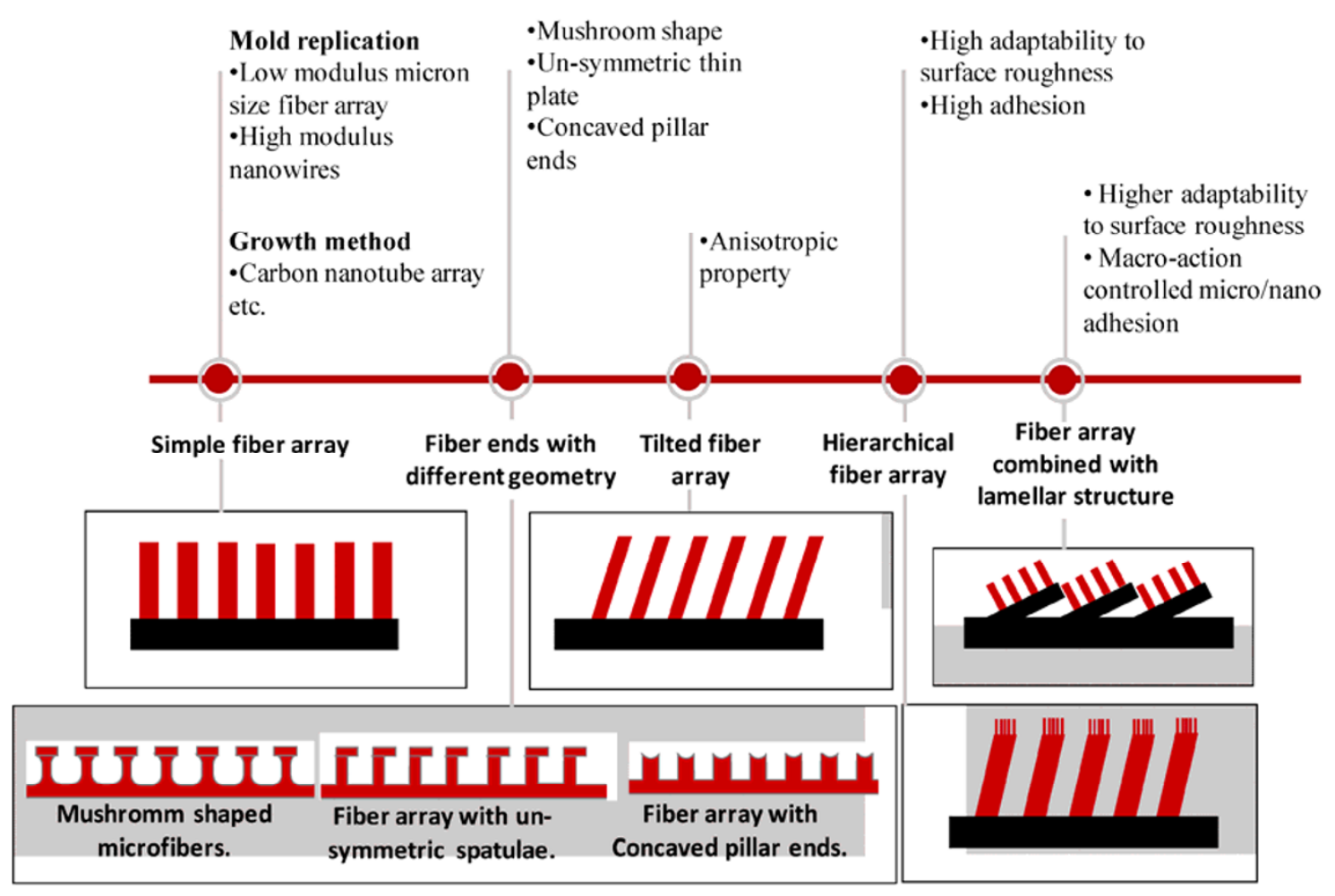

Fig. 4 Fabrication strategies of gecko-inspired surfaces.

shows the potential applications of nanowires and nanotubes with a high Young's modulus [101].

Fiber arrays with different end shapes, such as mushrooms, asymmetric spatulae, and concave structures, have been reported [97, 102, 103]. In practice, the mushroom-shaped fiber array is the most commonly used [104-108]. Gorb et al. [72] reported a geckoinspired mushroom surface made of polyvinyl siloxane (PVS) with an adhesion strength of approximately $50 \mathrm{kPa}$. Kim and Sitti fabricated a fibrillar mushroom surface with polyurethane (PU) that generated $180 \mathrm{kPa}$ [109], and Davies reported that a fibrillar poly(dimethylsiloxane) (PDMS) surface can reach $219 \mathrm{kPa}$ [106]. A combination of lithography and the two-step molding process is now also widely used for fabricating hierarchical fibrillar surfaces [37, 110-112]. Deep reactive ion etching [113], self-assembly [114], anodic oxidation [113], angled etching, and mechanical yielding techniques or methods [37, 115-117] have also been explored to fabricate templates of gecko-inspired dry adhesive surfaces.

The incorporation of hierarchical structures into fibers has also been explored. Two- or three-level fiber arrays produce higher adhesion strength than singlelevel ones [105, 118-121]. Jeong et al. [86] reported that the adhesion of two-level fiber arrays made of polyurethane acrylate does not decrease with an increase in surface roughness as long as it is less than $20 \mu \mathrm{m}$, exhibiting better adaptabilities than a singlelevel fiber array. This gecko-inspired surface generated an adhesion strength of $260 \mathrm{kPa}$, which can be used for moving on large-area glass surfaces [86].

The upper supporting level that mimics the lamella or foot is usually fabricated as the backing layer of the fiber array [122-124]. Lee et al. [122] fabricated a gecko-inspired polyethylene surface, combining lamellae and nanofiber arrays by heat rolling, which exhibited high compliance. Tian et al. [125] experimentally revealed that the soft lamellar skin of the gecko acts as a soft spring and contributes to the reliable control of a wide range of adhesive states rather than a repulsive state. Further, the three-legged hybrid clamp mimicking a lamellar skin/setae structure was developed to transfer a horizontally placed silicon wafer. Sameoto et al. [123] fabricated a surface that combines the macroscale substrate and the fiber array 
to increase adaptability. Northen et al. [124] reported a method to actively switch between adhesion and non-adhesion by controlling the orientation of the cantilever by a magnetic field; the adhesion strength was only $14 \mathrm{~Pa}$, but provided the general proof-ofconcept that adhesion can be reversibly controlled through an external stimulus.

A selection of materials for gecko-inspired adhesives, such as polymide [89, 106], PVS [25, 74], PDMS [99, 106, 108, 126], poly(methyl methacrylate) (PMMA) [115], polyurethane [109-110, 113], polystyrene (PS) [105, 114], silicon rubber [127], polypropylene [128], and polyethylene has also been considered [122]. It is proposed that polyurethane with a low Young's modulus can generate strong adhesion because the polar groups contribute to the enhancement of the adhesion; thus, this material may be suitable for gecko-inspired adhesives [129]. Lee et al. [130] coated the fabricated pillars with a mussel-adhesive-protein-mimetic polymer in order to improve the reversible wet adhesion property under water. This chemical coating method appears to be effective in enhancing the adhesion of functional surfaces.

Since the thermal and electric properties of polymer materials are not satisfactory in certain applications, aligned carbon nanotubes with stable electrical and thermal properties have drawn increased attention [124, 131-134]. Yurdumankan et al. [132, 133] first reported a multiwalled carbon nanotube (MWCNT) adhesive with a PMMA-backing layer in which the adhesion stress reached $16 \mathrm{MPa}$ based on experiments conducted on the nanoscale. CNT arrays with an adhesion strength of $110 \mathrm{kPa}$ have been achieved by Zhao et al. [134], but their durability is poor. Ge et al. $[135,136]$ reported that the CNT array supports a 200-kPa shear stress over a period of 8-12 h without any cohesive break; the adhesion strength was 30$50 \mathrm{kPa}$. A large increase in the adhesion and friction strength of CNT adhesives was also reported by $\mathrm{Qu}$ et al. [137, 138] who achieved $100 \mathrm{kPa}$ and $1 \mathrm{MPa}$, respectively. Fiber arrays with a high Young's modulus can generate strong adhesion because of the compliance of nanotubes and their strong van der Waals forces [34, 139-141].

\subsubsection{Anisotropic friction of gecko-inspired surfaces}

The tribological properties of materials mainly rely on the surface structures and the chemical nature of the surfaces. Anisotropy exists widely in nature [142]. At the macroscopic scale, for example, textured structures are widely applied in the weaving industry, sole decorative patterns, tires, and roads in the form of friction anisotropy. Friction anisotropy also exists between crystalline surfaces, such as mica, exfoliated graphene, synthetic self-assembled monolayers, and quasi-crystalline structures of metallic alloys, which are assigned to the lattice structure or elastic puckering. The skin of several animals also have anisotropic structures, such as the feathers of birds, the scales of fish and snakes, and the finer hierarchical micro- and nano-scale structures of lizards, geckos, and flies. Among these structures, the gecko with its hierarchical structures from the macro- to the micro- and nanoscales shows great advantages in terms of anisotropic friction and adhesion properties, which enable geckos to rapidly switch between attachment and detachment on both walls (requiring switchable friction) and ceilings (requiring switchable adhesion) [22].

Two types of structures can be used for fabricating anisotropic gecko-inspired adhesives: inclined and asymmetric structures. According to the fibrillar adhesion models, an inclined fiber array generates friction anisotropy [116, 143, 144]. For example, Murphy et al. [135] prepared an angled spatula-shaped fibrillar surface that generated obvious anisotropic friction and adhesion. The adhesion force along the inclined direction of the inclined fiber array produced by $\mathrm{Yu}$ et al. [116] was 6 to 7 times higher than that along the inclined direction. Zhou et al. [139] posited that inclined MWCNT array surfaces produce stable friction anisotropy over several thousands of cycles. Fiber arrays with asymmetric shapes or asymmetric spatulae at the ends also produce frictional anisotropy [88, 108, 145-147]. Yoon et al. [89] reported on Janusfaced fiber arrays by selectively depositing a metal layer only on one side, leading to friction anisotropies.

\section{Future research avenues}

Although recently there have been significant advances in modeling the friction and adhesion mechanisms of geckos, some challenging issues remain to fully understand and mimic this complex frictional-adhesive 
system. The contribution of hierarchical structures is still not fully understood. The role of the lamellae has not been extensively explored. The experiments on a single seta by Autumn et al. showed that sliding a single seta on a surface at a distance of approximately $5 \mu \mathrm{m}$ can maximize the adhesion force while a larger distance is required for the setal array. There are still no models that can fully explain the anisotropic mechanical deformations of the hierarchical structures and the peel processes during sliding. The articulation and deformations of all the different structures during gripping and releasing is another future research direction. Thus, the hierarchical design principles of the gecko-inspired dry adhesive surfaces have yet to be fully identified and established.

Further, the effects of the sliding velocity on gecko adhesion and friction have not been fully explored, although a few reports are available [148]. Most friction models are based on the Coulomb Law or Model of Friction, which may not apply to fine biological structures. The interfacial interactions between the foot pad proteins and the substrate surfaces must be further investigated in order to fully understand the impact of the sliding velocity on adhesion and friction forces. The stick-slip phenomena also need to be studied further [148].

Gecko-inspired adhesive surfaces have been proposed for various applications, such as wall-climbing robots, reversible self-adhesive labels, fixation and fastening, and biomedical materials and sports equipment, which require remarkable properties, including reversible attachment and detachment without breakage, strong stability in a wide range of humidity and temperature, and high strength and easy (low energy) motion during adhesion. Further work resolving these issues will no doubt allow us to realize the full potential for the applications of gecko-inspired adhesive surfaces.

\section{Acknowledgments}

Z.M. and Y.T. are supported by the Natural Science Foundation of China (Grant Nos. 51175281 and 51021064). H.Z. acknowledges the support of the University of Alberta China Opportunity Fund and a Discovery Grant Award from the Natural Sciences and Engineering Research Council of Canada (NSERC).
N.P. acknowledges support through a PFund grant from the Louisiana Board of Regents. J.N.I.'s contribution to parts of this review was sponsored by the UCSB Institute for Collaborative Biotechnologies Grant W911NF-09-D-000 from the U.S. Army Research Office (the content of the information does not necessarily reflect the position or the policy of the Government, and no official endorsement should be inferred).

Open Access: This article is distributed under the terms of the Creative Commons Attribution Noncommercial License which permits any noncommercial use, distribution, and reproduction in any medium, provided the original author(s) and source are credited.

\section{References}

[1] Autumn K, Hsieh S T, Dudeket D M, Chen J, Chitaphan C, Full R J. Dynamics of geckos running vertically. J Exp Biol 209: 260-272 (2006)

[2] Hansen W, Autumn K. Evidence for self-cleaning in gecko setae. P Natl Acad Sci USA 102: 385-389 (2005)

[3] Cutkosky M R. Gecko-like robot scampers up the wall. New Sci 2252: 29-33 (2006)

[4] Menon C, Murphy M, Sitti M. Gecko inspired surface climbing robots. In Proceedings of the IEEE International Conference on Robotics and Biomimetics, Shenyang, China, 2004: 431-436.

[5] Ruibal R, Ernst V. The structure of the digital setae of lizards. J Morphol 117: 271-294 (1965)

[6] Russell A P, Bauer A M, Laroiya R. Morphological correlates of the secondarily symmetrical Pes of Gekkotan lizards. $J$ Zool 241: 767-790 (1997)

[7] Autumn K. How gecko toes stick-The powerful, fantastic adhesive used by geckos is made of nanoscale hairs that engage tiny forces, inspiring envy among human imitators. Am Sci 94: 124-132 (2006)

[8] Autumn K, Peattie A M. Mechanisms of adhesion in geckos. Integr Comp Biol 42: 1081-1090 (2002)

[9] Autumn K, Sitti M, Peattie A, Liang A, Hansen W, Sponberg S, Kenny T, Fearing R, Israelachvili J, Full R. Evidence for van der Waals adhesion in gecko setae. P Natl Acad Sci USA 99: 12252-12256 (2002)

[10] Sun W X, Neuzil P, Kustandi T S, Oh S, Samper V D. The nature of the gecko lizard adhesive force. Biophys $J \mathbf{8 9}$ : L14-L17 (2005)

[11] Huber G, Mantz H, Spolenak R, Mecke K, Jacobs K, Gorb $\mathrm{S} N$, Arzt E. Evidence for capillarity contributions to gecko 
adhesion from single spatula nanomechanical measurements. P Natl Acad Sci Usa 102: 16293-16296 (2005)

[12] Prowse M S, Wilkinson M, Puthoff J B, Mayer G, Autumn K. Effects of humidity on the mechanical properties of gecko setae. Acta Biomater 7: 733-738 (2011)

[13] Kim T W, Bhushan B. The adhesion model considering capillarity for gecko attachment system. $J R$ Soc Interface 5: 319-327 (2008)

[14] Niederegger S, Gorb S N. Friction and adhesion in the tarsal and metatarsal scopulae of spiders. J Comp Physiol A 192: 1223-1232 (2006)

[15] Niewiarowski P H, Lopez S, Ge L H, Hagan E, Dhinojwala A. Sticky gecko feet: The role of temperature and humidity. PLoS One 3: e2192 (2008)

[16] Pesika N S, Zeng H B, Kristiansen K, Zhao B, Tian Y, Autumn K, Israelachvili J. Gecko adhesion pad: A smart surface? J Phys-Condens Mat 21: 464132 (2009).

[17] Puthoff J B, Prowse M S, Wilkinson M, Autumn K. Changes in materials properties explain the effects of humidity on gecko adhesion. J Exp Biol 213: 3699-3704 (2010)

[18] Autumn K, Liang Y A, Hsieh S T, Zesch W, Chan WP, Kenny T W, Fearing R, Full R J . Adhesive force of a single gecko foot-hair. Nature 405: 681-685 (2000)

[19] Huber G, Gorb S, Spolenak R, Arzt E. Resolving the nanoscale adhesion of individual gecko spatulae by atomic force microscopy. Biol Lett 1: 2-4 (2005)

[20] Autumn K, Dittmore A, Santos D, Spenko M, Cutkosky M. Frictional adhesion: A new angle on gecko attachment. $J$ Exp Biol 209: 3569-3579 (2006)

[21] Zhao B X, Pesika N, Rosenberg K, Tian Y, Zeng H, McGuiggan P, Autumn K, Israelachvili J. Adhesion and friction force coupling of gecko setal arrays: Implications for structured adhesive surfaces. Langmuir 24: 1517-1524 (2008)

[22] Wan J, Tian Y, Zhou M, Zhang X J, Meng Y G. Experimental research of load effect on the anisotropic friction behaviors of gecko seta array. Acta Phys Sin 61: 016202 (2012)

[23] Johnson K L, Kendall K, Roberts A D. Surface energy and contact of elastic solids. Proc R. Soc London Ser A 324: 301-313 (1971)

[24] Arzt E, Gorb S, Spolenak R. From micro to nano contacts in biological attachment devices. P Natl Acad Sci USA 100: 10603-10606 (2003)

[25] Peressadko A, Gorb S N. When less is more: Experimental evidence for tenacity enhancement by division of contact area. J Adhes 80: 247-261 (2004)

[26] Peattie A M, Full R J. Phylogenetic analysis of the scaling of wet and dry biological fibrillar adhesives. P Natl Acad Sci USA 104: 18595-18600 (2007)
[27] Autumn K, Hansen W. Ultrahydrophobicity indicates a non-adhesive default state in gecko setae. J Comp Physiol A 193: 1205-1212 (2006)

[28] Tang T, Hui C Y, Glassmaker N J. Can a fibrillar interface be stronger and tougher than a non-fibrillar one? $J R S o c$ Interface 2: 505-516 (2005)

[29] Bonser R. The Young's modulus of ostrich claw keratin. $J$ Mater Sci Lett 19: 1039-1040 (2000)

[30] Peattie A M, Majidi C, Corder A B, Full R J. Similar elastic modulus of setal keratin for two species of gecko. Integr Comp Biol 461: E109 (2006)

[31] Peattie A M, Majidi C, Corder A, Full R J. Ancestrally high elastic modulus of gecko setal beta-keratin. $J$ R Soc Interface 4: 1071-1076 (2007)

[32] Autumn K, Majidi C, Groff R E, Dittmore A, Fearing R. Effective elastice modulus of isolated gecko setal arrays. J Exp Biol 209: 3558-3568 (2006)

[33] Persson B. On the mechanism of adhesion in biological systems. J Chem Phys 118: 7614-7621 (2003)

[34] Schubert B, Majidi C, Groff R E, Baek S, Bush B, Maboudian R, Fearing R S. Towards friction and adhesion from high modulus microfiberarrays. J Adhes Sci Technol 21: 1297-1315 (2007)

[35] Kwak J S, Kim T W. A review of adhesion and friction models for gecko feet. Int J Precis Eng Manuf 11: 171-186 (2010)

[36] Campolo D, Jones S, Fearing R S. Fabrication of gecko foot-hair like nano structures and adhesion to random rough surfaces. In Proceedings of 2003 IEEE International Conference on Nanotechnology, SAN FRANCISCO, USA, 2003: 856-859.

[37] Aksak B, Murphy M P, Sitti M. Adhesion of biologically inspired vertical and angled polymer microfiber arrays. Langmuir 23: 3322-3332 (2007)

[38] Reyes M P, Fearing R S. Macromodel for the mechanics of gecko hair adhesion. In Proceedings of 2008 IEEE International Conference on Robotics and Automation, Pasadena, 2008: 1602-1607.

[39] Jagota A, Bennison S J. Mechanics of adhesion through a fibrillar microstructure. Integr Comp Biol 42: 1140-1145 (2002)

[40] Gao H J, Wang X, Yao H M, Gorb S, Arzt E. Mechanics of hierarchical adhesion structures of geckos. Mech Mater 37: 275-285 (2005)

[41] Yao H, Gao H. Mechanics of robust and releasable adhesion in biology: Bottom-up designed hierarchical structures of gecko. J Mech Phys Solids 54: 1120-1146 (2006)

[42] Yao H, Gao H. Bio-inspired mechanics of bottom-up designed hierarchical materials: Robust and releasable adhesion systems 
of gecko. Bull Pol Acad Sci-Tech 55: 141-150 (2007)

[43] Bhushan B, Peressadko A G, Kim T W. Adhesion analysis of two-level hierarchical morphology in natural attachment systems for 'smart adhesion'. J Adhes Sci Technol 20: 14751491 (2006)

[44] Kim T W, Bhushan B. Adhesion analysis of multi-level hierarchical attachment system contacting with a rough surface. J Adhes Sci Technol 21: 1-20 (2007)

[45] Kim T W, Bhushan B. Effect of stiffness of multi-level hierarchical attachment system on adhesion enhancement. Ultramicroscopy 107: 902-912 (2007)

[46] Zeng H B, Pesika N, Tian Y, Zhao B, Chen Y, Tirrell M, Turner K L, Israelachvili J N. Frictional adhesion of patterned surfaces and implications for gecko and biomimetic systems. Langmuir 25: 7486-7495 (2009)

[47] Shah G J, Sitti M. Modeling and design of biomimetic adhesives inspired by gecko foot-hairs. In Proceedings of the IEEE International Conference on Robotics and Biomimetics, Shenyang, 2004: 873-878.

[48] Peressadko A G, Gorb S N. Surface profile and friction force generated by insects. In Proceedings of the 1st International Industrial Conference Bionik 2004, Hannover, 2004: 257-261.

[49] Huber G, Gorb S N, Hosoda N, Spolenak R, Arzt E. Influence of surface roughness on gecko adhesion. Acta Biomater 3: 607-610 (2007)

[50] Kendall K. Thin-film peeling-The elastic term. J Phys D Appl Phys 8: 1449-1452 (1975)

[51] Peng Z L, Chen S H, Soh A K. Peeling behavior of a bio-inspired nano-film on a substrate. Int J Solids Struct 47: 1952-1960 (2010)

[52] Pesika N S, Tian Y, Zhao B X, Rosenberg K, Zeng H, McGuiggan P, Autumn K, Israelachvili J N. Peel-zone model of tape peeling based on the gecko adhesive system. $J$ Adhes 83: 383-401 (2007)

[53] Zhang L, Wang J. A generalized cohesive zone model of the peel test for pressure-sensitive adhesives. Int $J$ Adhes Adhes 29: 217-224 (2009)

[54] Lin Y Y, Hui C Y, Wang Y C. Modeling the failure of an adhesive layer in a peel test. J Polym Sci Pol Phys 40: 2277-2291 (2002)

[55] Zhou M, Tian Y, Pesika N, Zeng H, Wan J, Meng Y G, Wen S Z. The extended peel zone model: Effect of peeling velocity. $J$ Adhes 87: 1045-1058 (2011)

[56] Tian Y, Pesika N, Zeng H B, Rosenberg K, Zhao B, McGuiggan P, Autumn K, Israelachvili J. Adhesion and friction in gecko toe attachment and detachment. $P$ Natl Acad Sci USA 103: 19320-19325 (2006)

[57] Endlein T, Ji A, Samuel D, Yao N, Wang Z, Barnes W P, Federle W, Kappl M, Dai Z D. Sticking like sticky tape:
Tree frogs use friction forces to enhance attachment on overhanging surfaces. J Roy Soc Interface 10: 20120838 (2011)

[58] Fuller K, Tabor D. Effect of surface-roughness on adhesion of elastic solids. P Roy Soc Lond A Mat 345: 327-342 (1975)

[59] Briggs G, Briscoe B J. Effect of surface-topography on adhesion of elastic solids. J Phys D Appl Phys 10: 2453-2466 (1977)

[60] Persson B, Gorb S. The effect of surface roughness on the adhesion of elastic plates with application to biological systems. J Chem Phys 119: 11437-11444 (2003)

[61] Peng Z L, Chen S H. Effects of surface roughness and film thickness on the adhesion of a bio-inspired nanofilm. Phys Rev E 83: 051915 (2011)

[62] Sponberg S, Hansen W, Peattie A, Autumn K. Dynamics of isolated gecko setal arrays. Am Zool 41: 1594-1594 (2001)

[63] Schargott M, Popov V L, Gorb S. Spring model of biological attachment pads. $J$ Theor Biol 243: 48-53 (2006)

[64] Wang Z Y, Gu W H, Wu Q, Ji A, Dai Z D. Morphology and reaction force of toes of geckos freely moving on ceilings and walls. Sci China Technol Sci 53:1688-1693 (2010)

[65] Zhao B X, Pesika N, Zeng H B, Wei W, Chen Y, Autumn K, Turner K, Israelachvili J. Role of tilted adhesion fibrils (setae) in the adhesion and locomotion of gecko-like systems. J Phys Chem B 113: 3615-3621 (2009)

[66] Wan J, Tian Y, Zhou M, Zhang X-J, Meng Y-G. Experimental research of load effect on the anisotropic friction behaviors of gecko seta array. Acta Phys Sin 61: 016202 (2012)

[67] Hill G, Soto D, Peattie A, Full R J, Kenny T W. Orientation angle and the adhesion of single gecko setae. $J$ Roy Soc Interface 8: 926-933 (2011)

[68] Pesika N, Gravish N, Wikinson M, Zhao B, Zeng H, Tian Y, Israelachvili J, Autumn K. The crowding model as a tool to understand and fabricate gecko-inspired dry adhesives. $J$ Adhes 85: 512-525 (2009)

[69] Filippov A, Popov V L, Gorb S N. Shear induced adhesion: Contact mechanics of biological spatula-like attachment devices. J Theor Biol 276: 126-131 (2011)

[70] Kumar A, Hui C Y. Numerical study of shearing of a microfibre during friction testing of a microfibre array. $P$ Roy Soc Lond A Mat 467: 1372-1389 (2011)

[71] Cheng Q H, Chen B, Gao H J, Zhang Y W. Sliding-induced non-uniform pretension governs robust and reversible adhesion: A revisit of adhesion mechanisms of geckos. $J$ Roy Soc Interface 9: 283-291 (2012)

[72] Chen B, Wu P D, Gao H J. Pre-tension generates strongly reversible adhesion of a spatula pad On substrate. J Roy Soc Interface 6: 529-537 (2009)

[73] Boesel L F, Greiner C, Arzt E, del Campo A. Gecko-inspired 
surfaces: A path to strong and reversible dry adhesives. $A d v$ Mater 22: 2125-2137 (2010)

[74] Gorb S, Varenberg M, Peressadko A, Tuma J. Biomimetic mushroom-shaped fibrillar adhesive microstructure. J Roy Soc Interface 4: 271-275 (2007)

[75] Stork N E. Experimental-analysis of adhesion of chrysolinapolita (chrysomelidae, coleoptera) on a variety of surfaces. J Exp Biol 88: 91-107 (1980)

[76] Chan E P, Greiner C, Arzt E, Crosby A J. Designing model systems for enhanced adhesion. Mrs Bull 32: 496-503 (2007)

[77] Kroner E, Arzt E. What we can learn from geckos. Nachr Chem 57: 137-139 (2009)

[78] Varenberg M, Peressadko A, Gorb S, Arzt E. Effect of real contact geometry on adhesion. Appl Phys Lett 89: 121905 (2006)

[79] Gao H J, Ji B H, Jager I L, Arzt E, Fratzl P. Materials become insensitive to flaws at nanoscale: Lessons from nature. P Natl Acad Sci USA 100: 5597-5600 (2003)

[80] Varenberg M, Murarash B, Kligerman Y, Gorb S N. Geometry-controlled adhesion: Revisiting the contact splitting hypothesis. Appl Phys A-Mater 103: 933-938 (2011)

[81] Spolenak R, Gorb S, Gao H J, Arzt E. Effects of contact shape on the scaling of biological attachments. P Roy Soc Lond A Mat 461: 305-319 (2005)

[82] Gorb S N, Varenberg M. Mushroom-shaped geometry of contact elements in biological adhesive systems. $J$ Adhes Sci Technol 21: 1175-1183 (2007)

[83] Varenberg M, Gorb S. Close-up of mushroom-shaped fibrillar adhesive microstructure: Contact element behaviour. $J$ Roy Soc Interface 5: 785-789 (2008)

[84] Creton C, Gorb S. Sticky feet: From animals to materials. Mrs Bull 32: 466-472 (2007)

[85] Gorb S N. Biological attachment devices: Exploring nature's diversity for biomimetics. Philos T R Soc A 366: 1557-1574 (2008)

[86] Geim A K, Dubonos S V, Grigorieva I V, Novoselov K S, Zhukov A A, Shapoval S Y. Microfabricated adhesive mimicking gecko foot-hair. Nat Mater 2: 461-463 (2003)

[87] Jeong H E, Lee J K, Kim H N, Moon S H, Suh K Y. A nontransferring dry adhesive with hierarchical polymer nanohairs. P Natl Acad Sci USA 106: 5639-5644 (2009)

[88] Parness A, Soto D, Esparza N, Gravish N, Wilkinson M, Autumn K, Cutkosky M. A microfabricated wedge-shaped adhesive array displaying gecko-like dynamic adhesion, directionality and long lifetime. J Roy Soc Interface 6: 1223-1232 (2009)

[89] Yoon H, Jeong H E, Kim T I, Kang T JAuthor Vitae, Tahk DAuthor Vitae, Char K, Author VSuh K Y. Adhesion hysteresis of janus nanopillars fabricated by nanomolding and oblique metal deposition. Nano Today 4: 385-392 (2009)

[90] Sitti M, Fearing R S. Synthetic gecko foot-hair micro/ nano-structures as dry adhesives. J Adhes Sci Technol 17: 1055-1073 (2003)

[91] Glassmaker N J, Jagota A, Hui C Y, Kim J. Design of biomimetic fibrillar interfaces: 1. Making contact. $J$ Roy Soc Interface 1: 23-33 (2004)

[92] Hui C Y, Jagota A, Lin Y Y, Kramer E J. Constraints on microcontact printing imposed by stamp deformation. Langmuir 18: 1394-1407 (2002)

[93] Spolenak R, Gorb S, Arzt E. Adhesion design maps for bioinspired attachment systems. Acta Biomater 1: 5-13 (2005)

[94] Greiner C, Spolenak R, Arzt E. Adhesion design maps for fibrillar adhesives: The effect of shape. Acta Biomaterialia 5: 597-606 (2009)

[95] Zhou M, Pesika N, Zeng H, Wan J, Zhang X, Meng Y, Wen $\mathrm{S}$, Tian Y. Design of gecko-inspired fibrillar surfaces with strong attachment and easy-removal properties: A numerical analysis of peel-zone. J Roy Soc Interface 9: 2424-2436 (2012)

[96] Crosby A J, Hageman M, Duncan A. Controlling polymer adhesion with "pancakes". Langmuir 21: 11738-11743 (2005)

[97] Campo D A, Greiner C. Su-8: A photoresist for high-aspectratio and 3D submicron lithography. J Micromech Microeng 17: R81-R95 (2007)

[98] Lamblet M, Verneuil E, Vilmin T, Buguin A, Silberzan P, Léger L. Adhesion enhancement through micropatterning at polydimethylsiloxane-acrylic adhesive interfaces. Langmuir 23: 6966-6974 (2007)

[99] Greiner C, Campo D A, Arzt E. Adhesion of bioinspired micropatterned surfaces: Effects of pillar radius, aspect ratio, and preload. Langmuir 23: 3495-3502 (2007)

[100] Lu G W, Hong W J, Tong L, Bai H, Wei Y, Shi G. Drying enhanced adhesion of polythiophene nanotubule arrays on smooth surfaces. ACS Nano 2: 2342-2348 (2008)

[101] Lee J, Fearing R S. Contact self-cleaning of synthetic gecko adhesive from polymer microfibers. Langmuir 24: 10587 10591 (2008)

[102] Campo D A, Greiner C, Arzt E. Contact shape controls adhesion of bioinspired fibrillar surfaces. Langmuir 23: 10235-10243 (2007)

[103] Campo D A, Greiner C, Alvarez I, Arzt E. Patterned surfaces with pillars with controlled 3D tip geometry mimicking bio-attachment devices. Adv Mater 19: 1973-1977 (2007)

[104] Aksak B, Murphy M P, Sitti M. Gecko inspired microfibrillar adhesives for wall climbing robots on micro/ nanoscale rough surfaces. In Proceedings of 2008 IEEE International Conference on Robotics and Automation, Pasadena, 2008: 3058-3063. 
[105] Lee D Y, Lee D H, Lee S G, Cho K. Hierarchical geckoinspired nanohairs with a high aspect ratio induced by nanoyielding. Soft Matter 8: 4905-4910 (2012)

[106] Davies J, Haq S, Hawke T, Sargent J P. A practical approach to the development of a synthetic gecko tape. Int $J$ Adhes Adhes 29: 380-390 (2009)

[107] Haefliger D, Boisen A. Three-dimensional microfabrication in negative resist using printed masks. J Micromech Microeng 16: 951-957 (2006)

[108] Sameoto D, Menon C. Direct molding of dry adhesives with anisotropic peel strength using an offset lift-off photoresist mold. J Micromech Microeng 19: 1-5 (2009)

[109] Kim S, Sitti M. Biologically inspired polymer microfibers with spatulate tips as repeatable fibrillar adhesives. Appl Phys Lett 89: 261911 (2006)

[110] Jin K, Tian Y, Erickson J S, Puthoff J, Autumn K, Pesika N S. Design and fabrication of gecko-inspired adhesives. Langmuir 28: 5737-5742 (2012)

[111] Sameoto D, Menon C. Deep UV patterning of acrylic masters for molding biomimetic dry adhesives. $J$ Micromech Microeng 20: 115037 (2010)

[112] Krahn J, Sameoto D, Menon C. Controllable biomimetic adhesion using embedded phase change material. Smart Mater Struct 20: 015014 (2011)

[113] Kim S, Sitti M, Hui C Y, Long R, Jagota A. Effect of backing layer thickness on adhesion of single-level elastomer fiber arrays. Appl Phys Lett 91: 161905 (2007)

[114] Kustandi T S, Samper V D, Yi D K, Ng W S, Neuzil P, Sun W. Self-assembled nanoparticles based fabrication of gecko foot-hair-inspired polymer nanofibers. Adv Funct Mater 17: 2211-2218 (2007)

[115] Yu J, Chary S, Das S, Tamelier J, Pesika N S, Turner K L, Israelachvili J N. Gecko-inspired dry adhesive for robotic applications. Adv Funct Mater 21: 3010-3018 (2011)

[116] Reddy S, Arzt E, Campo D A. Bioinspired surfaces with switchable adhesion. Adv Mater 19: 3833-3837 (2007)

[117] Sato H, Houshi Y, Shoji S. Three-dimensional microstructures consisting of high aspect ratio inclined micro-pillars fabricated by simple photolithography. Microsyst Technol 10: 440-443 (2004)

[118] Kustandi T S, Samper V D, Ng W S, Chong A S, Gao H. Fabrication of a gecko-like hierarchical fibril array using a bonded porous alumina template. J Micromech Microeng 17: N75-N81 (2007)

[119] Greiner C, Arzt E, Campo D A. Hierarchical gecko-like adhesives. Adv Mater 21: 479-482 (2009)

[120] Murphy M P, Kim S, Sitti M. Enhanced adhesion by gecko-inspired hierarchical fibrillar adhesives. ACS Appl Mater Inter 1: 849-855 (2009)
[121] Bhushan B, Lee H. Fabrication and characterization of multi-level hierarchical surfaces. Faraday Discuss 156: 235-241 (2012)

[122] Lee J, Bush B, Maboudian R, Fearing R S. Gecko-inspired combined lamellar and nanofibrillar array for adhesion on nonplanar surface. Langmuir 25: 12449-12453 (2009)

[123] Sameoto D, Li Y S, Menon C. Multi-scale compliant foot designs and fabrication for use with a spider-inspired climbing robot. J Bionic Eng 5: 189-196 (2008)

[124] Northen M T, Greiner C, Arzt E, Turner K L. A geckoinspired reversible adhesive. Adv Mater 20: 3905-3909 (2008)

[125] Tian Y, Wan J, Pesika N, Zhou M. Bridging nanocontacts to macroscale gecko adhesion by sliding soft lamellar skin supported setal array. Sci Rep 3: 1382 (2013)

[126] Menon C, Murphy M, Sitti M. Gecko inspired surface climbing robots. In Proceedings of 2008 IEEE International Conference on Robotics and Automation, Pasadena, 2004: 3058-3063.

[127] Sitti M, Fearing R S. Nanomolding based fabrication of synthetic gecko foot-hairs. In Proceedings of the 2002 2nd IEE Conference on Nanotechnology, Washington, 2002: 137-140.

[128] Lee J, Fearing R S. Contact self-cleaning of synthetic gecko adhesive from polymer microfibers. Langmuir 24: 1058710591 (2008)

[129] Dai Z D, Yu M, Gorb S. Adhesion characteristics of polyurethane for bionic hairy foot. J Intel Mat Syst Str 17: 737-741 (2006)

[130] Lee H, Lee B P, Messersmith P B. A reversible wet/dry adhesive inspired by mussels and geckos. Nature 448: 334-338 (2007)

[131] Ajayan P M, Zhou O Z. Applications of carbon nanotubes. In Carbon Nanotubes. Berlin: Springer, 2001: 391-425.

[132] Yurdumakan B, Raravikar N R, Ajayan P M, Dhinojwala A. Synthetic gecko foot-hairs from multiwalled carbon nanotubes. Chem Commun: 3799-3801 (2005)

[133] Dhinojwala A, Ge L H, Sethi S, Yurdumakan B, Lijie C, Ajayan P M. Synthetic gecko foot-hairs from multiwalled carbon nanotubes. In 2007 Spring National ACS Meeting, Chicago, 2007: 64.

[134] Zhao Y, Tong T, Delzeit L, Kashani A, Meyyappan M, Majumdar A. Interfacial energy and strength of multiwalledcarbon-nanotube-based dry adhesive. J Vac Sci Technol B 24: 331-335 (2006)

[135] Wirth C T, Hofmann S, Robertson J. Surface properties of vertically aligned carbon nanotube arrays. Diam Relat Mater 17: 1518-1524 (2008)

[136] Ge L H, Sethi S, Ci L, Ajayan P M, Dhinojwala A. Carbon 
nanotube-based synthetic gecko tapes. P Natl Acad Sci USA 104: 10792-10795 (2007)

[137] Qu L, Dai L. Gecko-foot-mimetic aligned single-walled carbon nanotube dry adhesives with unique electrical and thermal properties. Adv Mater 19: 3844-3849 (2007)

[138] Qu L, Dai L, Stone M, Xia Z, Wang Z L. Carbon nanotube arrays with strong shear binding-on and easy normal liftingoff. Science 322: 238-242 (2008)

[139] Majidi C S, Groff R E, Fearing R S. Attachment of fiber array adhesive through side contact. J Appl Phys 98: 103521 (2005)

[140] Zhou M, Liu K, Wan J, Li X, Jiang K, Zeng H, Zhang X, Meng Y G, Shizhu Wen S Z, Zhu H W, Tian Y. Anisotropic interfacial friction of inclined multiwall carbon nanotube array surface. Carbon 30: 5372-5379 (2012)

[141] $\mathrm{Hu} \mathrm{S} \mathrm{H}$, Xia Z H, Gao X S. Strong adhesion and friction coupling in hierarchical carbon nanotube arrays for dry adhesive applications. ACS Appl Mater Inter 4: 1972-1980 (2012)

[142] Zheng Y M, Gao X F, Jiang L. Directional adhesion of superhydrophobic butterfly wings. Soft Matter 3: 178-182 (2007)

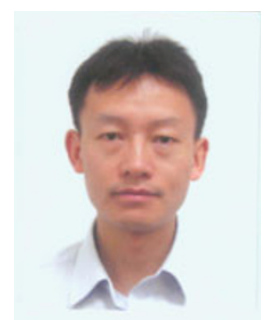

Yu TIAN, born 1975, is the Professor and Associate Director of the State Key Laboratory of Tribology at Tsinghua University in China. Tian gained his $\mathrm{BA}$ and $\mathrm{PhD}$ in Mechanical Engineering at Tsinghua University in 1998 and 2002, respectively. Then he became a faculty of the State Key Laboratory of Tribology at Tsinghua University. He did his postdoc at the University of California, Santa Barbara with Professor Jacob Israelachvili (from 2005 to 2007). He has been a visiting associate

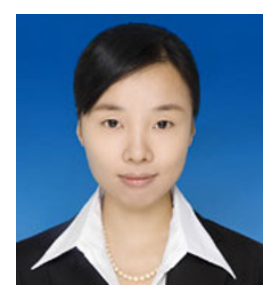

Ming ZHOU, graduated in 2007 from Tsinghua University, and received her $\mathrm{PhD}$ degree in 2013 from the SKLT, Tsinghua University. Now she is working as an engineer in the Institute of Mechanical Manufacturing
[143] Lee J H, Fearing R S, Komvopoulos K. Directional adhesion of gecko-inspired angled microfiber arrays. Appl Phys Lett 93: 191910 (2008)

[144] Murphy M P, Aksak B, Sitti M. Gecko-inspired directional and controllable adhesion. Small 5: 170-175 (2009)

[145] Tamelier J, Chary S, Turner K L. Vertical anisotropic microfibers for a gecko-inspired adhesive. Langmuir 28: 8746-8752 (2012)

[146] Sameoto D, Menon C. A low-cost, high-yield fabrication method for producing optimized biomimetic dry adhesives. J Micromech Microeng 19: 115002 (2009)

[147] Shon J K, Kong S S, Kim J M, Ko C H, Jin M, Lee Y Y, Hwang S H, Yoon J A, Kim J N. Facile synthesis of highly ordered mesoporous silver using cubic mesoporous silica template with controlled surface hydrophobicity. Chem Commun 14: 650-652 (2009)

[148] Gravish N, Wilkinson M, Sponberg S, Parness A, Esparza N, Soto D, Yamaguchi T, Broide M, Cutkosky M, Creton C, Autumn K. Rate-dependent frictional adhesion in natural and synthetic gecko setae. J Roy Soc Interface 7: 259-269 (2010)

professor at Nanyang Technology University for five months. His research interest is the science and technology at the interface of physics, materials, engineering and biology to understand the physical laws of adhesion, friction and rheology. He has published over 70 peer-reviewed journal papers. He has received the Wen Shizhu-Maple Award-Young Scholar award (2012), the Young Scholar Achievement Award of the Society of Mechanical Engineering of China (2011), Outstanding Young Scholar Award of the Chinese Tribology Institute (2009), and the National Excellent Doctoral Dissertation of China (2004).

Technology, China Academy of Engineering Physics. She has published 9 papers. Her research interests include the mechanism and application of gecko adhesion and the gecko-inspired surfaces, nano contact mechanics, nano-tribology, and more recently, the techniques and mechanisms of ultra-precision machining. 